\title{
Ventilation with lower tidal volumes as compared with conventional tidal volumes for patients without acute lung injury: a preventive randomized controlled trial
}

Rogier M Determann1,2, Annick Royakkers 3,4, Esther K Wolthuis',5, Alexander P Vlaar1, Goda Choi1,2, Frederique Paulus ${ }^{1}$, Jorrit-Jan Hofstra ${ }^{1,4}$, Mart J de Graaff', Johanna C Korevaar6 and Marcus J Schultz*1,7

\begin{abstract}
Introduction: Recent cohort studies have identified the use of large tidal volumes as a major risk factor for development of lung injury in mechanically ventilated patients without acute lung injury (ALI). We compared the effect of conventional with lower tidal volumes on pulmonary inflammation and development of lung injury in critically ill patients without ALI at the onset of mechanical ventilation.

Methods: We performed a randomized controlled nonblinded preventive trial comparing mechanical ventilation with tidal volumes of $10 \mathrm{ml}$ versus $6 \mathrm{ml}$ per kilogram of predicted body weight in critically ill patients without ALI at the onset of mechanical ventilation. The primary end point was cytokine levels in bronchoalveolar lavage fluid and plasma during mechanical ventilation. The secondary end point was the development of lung injury, as determined by consensus criteria for ALI, duration of mechanical ventilation, and mortality.
\end{abstract}

Results: One hundred fifty patients (74 conventional versus 76 lower tidal volume) were enrolled and analyzed. No differences were observed in lavage fluid cytokine levels at baseline between the randomization groups. Plasma interleukin-6 (IL-6) levels decreased significantly more strongly in the lower-tidal-volume group ((from 51 (20 to 182) $\mathrm{ng} / \mathrm{ml}$ to 11 (5 to 20$) \mathrm{ng} / \mathrm{ml}$ versus 50 (21 to 122$) \mathrm{ng} / \mathrm{ml}$ to 21 (20 to 77$) \mathrm{ng} / \mathrm{ml} ; P=0.01)$ ). The trial was stopped prematurely for safety reasons because the development of lung injury was higher in the conventional tidal-volume group as compared with the lower tidal-volume group (13.5\% versus $2.6 \% ; P=0.01)$. Univariate analysis showed statistical relations between baseline lung-injury score, randomization group, level of positive end-expiratory pressure (PEEP), the number of transfused blood products, the presence of a risk factor for ALI, and baseline IL-6 lavage fluid levels and the development of lung injury. Multivariate analysis revealed the randomization group and the level of PEEP as independent predictors of the development of lung injury.

Conclusions: Mechanical ventilation with conventional tidal volumes is associated with sustained cytokine production, as measured in plasma. Our data suggest that mechanical ventilation with conventional tidal volumes contributes to the development of lung injury in patients without ALI at the onset of mechanical ventilation.

Trial registration: ISRCTN82533884

* Correspondence: m.j.schultz@amc.uva.nl

1 Department of Intensive Care Medicine, Academic Medical Center, Meibergdreef 9, 1105 AZ, Amsterdam, The Netherlands

See related commentary by Villar and Slutsky, http://ccforum.com/content/14/1/120

\section{Introduction}

Mechanical ventilation is a life-saving strategy in patients with acute respiratory failure. Nevertheless, unequivocal evidence from both experimental and clinical studies indicates that mechanical ventilation has the potential to aggravate lung injury [1-3]. Data from three randomized controlled clinical trials confirmed the existence of venti- 
lator-associated lung injury in patients with acute lung injury (ALI) or its more-severe form, acute respiratory distress syndrome (ARDS), by showing reduced morbidity and mortality in the lower tidal-volume arm [3-6]. As a result of these studies, current guidelines now clearly support the use of lower tidal volume in patients with ALI/ARDS [7]. In contrast, little evidence supports the use of lower tidal volume in critically ill patients without ALI/ARDS, partly because of a lack of randomized controlled trial evidence on the best ventilator strategies in these patients [8].

Pneumonia, aspiration, sepsis, trauma, shock, and multiple blood transfusions are well-described risk factors for ALI/ARDS [9]. Animal studies demonstrate that mechanical ventilation with conventional tidal volume not only may aggravate, but also may initiate lung injury $[1,2]$. The role of tidal-volume size as a contributor to the development of lung injury in humans is, however, less clear. One study on reduced tidal volume and pressure settings did not show a reduction in mortality but suggested more side effects of lower tidal-volume ventilation in patients at high risk for ALI/ARDS [10]. Conversely, pulmonary procoagulant changes and increased systemic cytokine production were observed in patients without preexisting lung injury receiving conventional-tidal-volume mechanical ventilation during surgery $[11,12]$.

Other studies have challenged these findings $[13,14]$. Results from cohort studies suggest that mechanical ventilation with conventional tidal volumes may cause or contribute to development of lung injury in critically ill patients who did not have ALI/ARDS at the onset of mechanical ventilation $[15,16]$. The inconclusive results from the studies in surgical patients may arise from the fact that these patients were ventilated for only a short period, whereas the patients of the larger cohort studies were critically ill patients who had been ventilated for a longer period.

As ALI/ARDS is characterized by a profound production of inflammatory mediators, it might be expected that if conventional tidal volumes contribute to development of lung injury, the injury also may be associated with increased production of cytokines. We therefore conducted a trial to determine whether mechanical ventilation with conventional or lower tidal volume would be associated with different cytokine patterns in the lungs and the plasma of critically ill patients without ALI at onset of mechanical ventilation. Secondary end points were development of lung injury, duration of mechanical ventilation, and mortality.

\section{Materials and methods}

\section{Participants}

From January 2005 until December 2007 patients were recruited in the intensive care departments of one academic and one regional teaching hospital in the Netherlands. The academic ICU is a 28-bed "closed format" department where medical/surgical patients (including neurosurgery/neurology, cardiothoracic surgery, and cardiology patients) were under the direct care of the ICU team. The ICU team comprised 10 full-time ICU physicians, eight subspecialty fellows, 12 residents, and occasionally one intern. The regional teaching ICU is an eightbed "open format" department with medical/surgical patients (not including neurosurgery and cardiothoracic surgery patients). The ICU team comprised three fulltime ICU physicians, five physicians who participate in evening and night shifts, and one resident. The two ICUs had similar standards of practice in terms of mechanicalventilation and sedation protocols.

Patients were eligible for the study if they did not meet the consensus criteria for ALI/ARDS [17] and needed mechanical ventilation for an anticipated duration of more than 72 hours. Patients had to be randomized less than 36 hours after the onset of mechanical ventilation. Exclusion criteria were age younger than 18 years, participation in other clinical trials, pregnancy, increased uncontrollable intracranial pressure, chronic obstructive pulmonary disease (defined as a forced expiratory volume in 1 second to a forced vital capacity ratio less than 0.64 and daily medication), restrictive pulmonary disease (evidence of chronic interstitial infiltration on chest radiograph), use of immunosuppressive agents (100 mg hydrocortisone per day was allowed), pulmonary thromboembolism, previous pneumectomy or lobectomy, and previous randomization in this study. Randomization was performed by using sealed opaque envelopes in blocks of 50 patients. Each study center had its own randomization block. The protocol was approved by the medical ethics committees of both hospitals, and written informed consent was obtained from the patient or closest relatives before entry in the study. All procedures were done in compliance with the Helsinki declaration.

\section{Interventions}

The volume-controlled mode was used for mechanical ventilation. To calculate tidal volume, predicted body weight was used, as described [3]. The target tidal volume in the conventional group was $10 \mathrm{ml} / \mathrm{kg}$ of predicted body weight, which was routine practice at the time of the conduct of the study. Patients from the intervention group were ventilated at tidal volumes of $6 \mathrm{ml} / \mathrm{kg}$ of predicted body weight. In case patients were randomized to $6 \mathrm{ml} /$ $\mathrm{kg}$, the attending physician was allowed to increase tidal- 
volume size to 7 to $8 \mathrm{ml} / \mathrm{kg}$ if patients had severe dyspnea, as identified by increased respiratory rate (more than 35 to 40 breaths per minute) accompanied by increasing levels of discomfort (with or without need for more sedation). Levels of PEEP were set, together with the level of inspired oxygen $\left(\mathrm{FiO}_{2}\right)$ depending of the $\mathrm{PaO}_{2}$ according to a local protocol.

The ventilator was routinely (3 times/day) switched to the pressure support mode. If the pressure support mode was tolerated, this mode was used for further mechanical ventilation. Toleration of pressure support mode was assessed at the discretion of the attending physician. The pressure support was adjusted to reach the target tidal volumes. In case the attending physician preferred pressure-support ventilation in a patient randomized to the lower-tidal-volume group, and the applied tidal volume exceeded the target tidal volume because of high levels of pressure support, then this was accepted. Such patients were kept in their original randomization group in the statistical analyses.

As soon as patients were ready to be weaned from the ventilator, the pressure-support level had to be lowered stepwise to $5 \mathrm{~cm} \mathrm{H}_{2} \mathrm{O}$ within 24 hours. If this was not possible because of severe dyspnea, then the pressure support had to be increased to maintain tidal-volume size based on randomization group. Attending physicians decided to extubate the patient, based on general extubation criteria (that is, responsive and cooperative, adequate oxygenation with $\mathrm{FiO}_{2}$ of $40 \%$ or less, hemodynamically stable, no uncontrolled arrhythmia, and having a rectal temperature greater than 36.0). If a patient had been weaned from the ventilator but was reintubated for additional mechanical ventilation within 28 days, the same tidal-volume protocol was resumed.

Lung injury was diagnosed if a patient met the consensus criteria [17]. If it was diagnosed by the attending physician, the local protocol mandated mechanical ventilation with a tidal volume of $6 \mathrm{ml} / \mathrm{kg}$ in a pressurecontrolled mode for the remaining ventilation period.

\section{Objective and outcomes}

The primary outcome was cytokine levels in blindly obtained bronchoalveolar lavage fluid and plasma. Development of lung injury (according to consensus criteria for ALI/ARDS) [17], duration of mechanical ventilation, and mortality were secondary outcomes.

\section{Data collection}

Demographic data, ventilation parameters, and clinical and radiologic data were recorded immediately after the ventilator settings were changed on day 0 . Each second day, ventilator settings, blood-gas parameters, radiographic data, and medication use were recorded until the patient was weaned from the ventilator. The oxygenation index was calculated as described earlier [18]. Mean airway pressure was measured with the ventilator. The lunginjury score (LIS) was calculated. On the day of enrollment and each second day until the patient was weaned from the ventilator, a bronchoalveolar minilavage was performed for the measurement of levels of tumor necrosis factor $\alpha$ (TNF- $\alpha)$, interleukin-1 $\beta$ (IL-1 $\beta)$, and interleukin-6 (IL-6). Simultaneously, blood samples were drawn from an indwelling arterial catheter for IL- 6 measurements. Minilavage was performed as described previously [19]. The recovered fluid was centrifuged at $1,500 \mathrm{~g}$ for 10 minutes at $4^{\circ} \mathrm{C}$. The supernatant was collected and stored at $-80^{\circ} \mathrm{C}$ until measurements were performed. All markers were measured with an enzyme-linked immunoassay (Sanquin, Amsterdam, The Netherlands).

\section{Definitions}

Sepsis was defined by the Bone criteria [20]. Septic shock was present in cases of persisted hypotension (mean, less than $60 \mathrm{~mm} \mathrm{Hg}$ ) despite fluid resuscitation or vasopressor use [20]. Pneumonia was diagnosed from new infiltrates on chest radiograph together with clinical signs of infection and positive sputum culture with no other explanation for the symptoms [21]. Chronic alcohol abuse was defined as a previously established diagnosis of chronic alcoholism, a prior admission for alcohol detoxification, or alcohol withdrawal [22].

\section{Sample size}

The power calculation was based on a previous study on ventilator-associated lung injury [23]. In this study, bronchoalveolar lavage fluid levels of IL- 6 increased by $\pm 20 \%$ in ALI/ARDS patients ventilated with a conventional regimen and decreased by $\pm 20 \%$ in patients ventilated with a protective regimen. Based on these differences and expected baseline IL-6 levels of $250 \mathrm{pg} / \mathrm{ml}$ [19], we calculated that to detect a difference in changes from baseline between groups of $100 \mathrm{pg} / \mathrm{ml}$, with a two-sided significance level of 0.05 and a power of $80 \%, 49$ patients had to be included in each group. As we studied patients without ALI/ARDS, we chose to study twice as many patients, resulting in a total of 200 patients.

\section{Lung injury diagnosis for interim analysis}

For reasons of safety, interim analyses on the development of lung injury were conducted after the inclusion of 100 and 150 patients. For this, all chest radiographs were reviewed by two independent physicians who were blinded to all clinical parameters and randomization groups. Any new or worsening abnormality was scored. Chest radiographs showing new or worsening abnormalities were selected for further review. During the review process, they had access to $\mathrm{PaO}_{2} / \mathrm{FiO}_{2}(\mathrm{P} / \mathrm{F})$, echocardiog- 


\begin{tabular}{lll}
\hline & $\begin{array}{l}\text { Conventional tidal volume group } \\
(\boldsymbol{n}=\mathbf{7 4 )}\end{array}$ & $\begin{array}{c}\text { Lower tidal volume group } \\
(\boldsymbol{n}=\mathbf{7 6})\end{array}$ \\
\hline Cardiac arrest & 22 & 32 \\
Neurologic disease & 24 & 15 \\
Sepsis & 7 & 4 \\
Pneumonia & 1 & 3 \\
Aspiration & -- & 1 \\
Trauma & 12 & 10 \\
Pancreatitis & -- & 1 \\
Medical other & 5 & 5 \\
Cardiopulmonary surgery & 1 & 3 \\
Other surgery & 2 & 2 \\
\hline
\end{tabular}

raphy, and fluid-balance data, pulmonary capillary wedge pressures (if measured), and the admission diagnosis. Both physicians were familiar with the consensus criteria for ALI/ARDS [17]. In case of disagreement, consensus had to be obtained while reviewing the patient together.

\section{Statistical analysis}

Data are presented as mean with standard deviation for parametric data or as medians with interquartile range (IQR) for nonparametric data. Baseline comparisons between groups were made with the Student $t$ test, Mann-Whitney $U$ test, $\chi^{2}$ test, or Fisher Exact test where appropriate. The Mann-Whitney $U$ test was used to compare baseline levels of cytokines between groups. To study the primary outcome, a linear mixed model was constructed on cytokine levels, adding time and randomization group as factors in the model. In this model, the interaction between time and randomization group was used to study differences over time between groups. If the residuals were not normally distributed in linear mixedmodel analyses, the data were transformed to the natural logarithm of the original data. The relation between cytokine levels and development of ALI/ARDS was studied with a multivariate logistic regression analysis. For the secondary outcome, development of ALI/ARDS was studied with the $X^{2}$ test. To show the incidence of ALI/ ARDS over time, a Kaplan-Meier curve was constructed, and the log-rank test was used to calculate differences between groups.

To study the effect of tidal volumes while correcting for risk factors for ALI, a multivariate logistic regression analysis was performed. Variables with a $P$ value $<0.10$ in univariate analysis were considered for a multivariate model. If collinearity between variables was found, then the weaker variables were removed from the multivariate model. A backward elimination method was used for the final model.

A two-tailed $P$ value $<0.05$ was considered to be statistically significant. Data were analyzed by using SPSS, version 14.02 (SPSS Inc., Chicago, IL).

\section{Results}

\section{Patients}

A flow diagram summarizing patient inclusion, allocation, and analysis is given in Figure 1 . At the second interim analysis, after 150 patients were included, the trial was stopped because more patients had developed lung injury in the conventional tidal-volume group as compared with the lower tidal-volume group ((10 patients (13.5\%) versus two patients $(2.6 \%) ; P=0.01)$ ). Demographics and admission diagnoses are shown in Tables 1 and 2. Study groups were well balanced with respect to the number of patients with $\mathrm{P} / \mathrm{F}$ less than 40 $\mathrm{kPa}$ and unilateral chest radiographs abnormalities, the number of patients with bilateral chest radiographs abnormalities but $\mathrm{P} / \mathrm{F}$ more than $40 \mathrm{kPa}$, and risk factors for ALI/ARDS. Patients randomized to the lower-tidalvolume group, however, tended to be older, and more patients were chronic smokers.

\section{Ventilation data}

Ventilator data are presented in Figure 2. Applied tidal volumes were lower in the lower-tidal-volume group as compared with the conventional-tidal-volume group at baseline after randomization $(6.4 \pm 1.0 \mathrm{ml} / \mathrm{kg}$ versus 10.0 $\pm 1.0 \mathrm{ml} / \mathrm{kg} ; P<0.001$ ), as was the maximum airway pres- 


\section{Table 1: Demographic data}

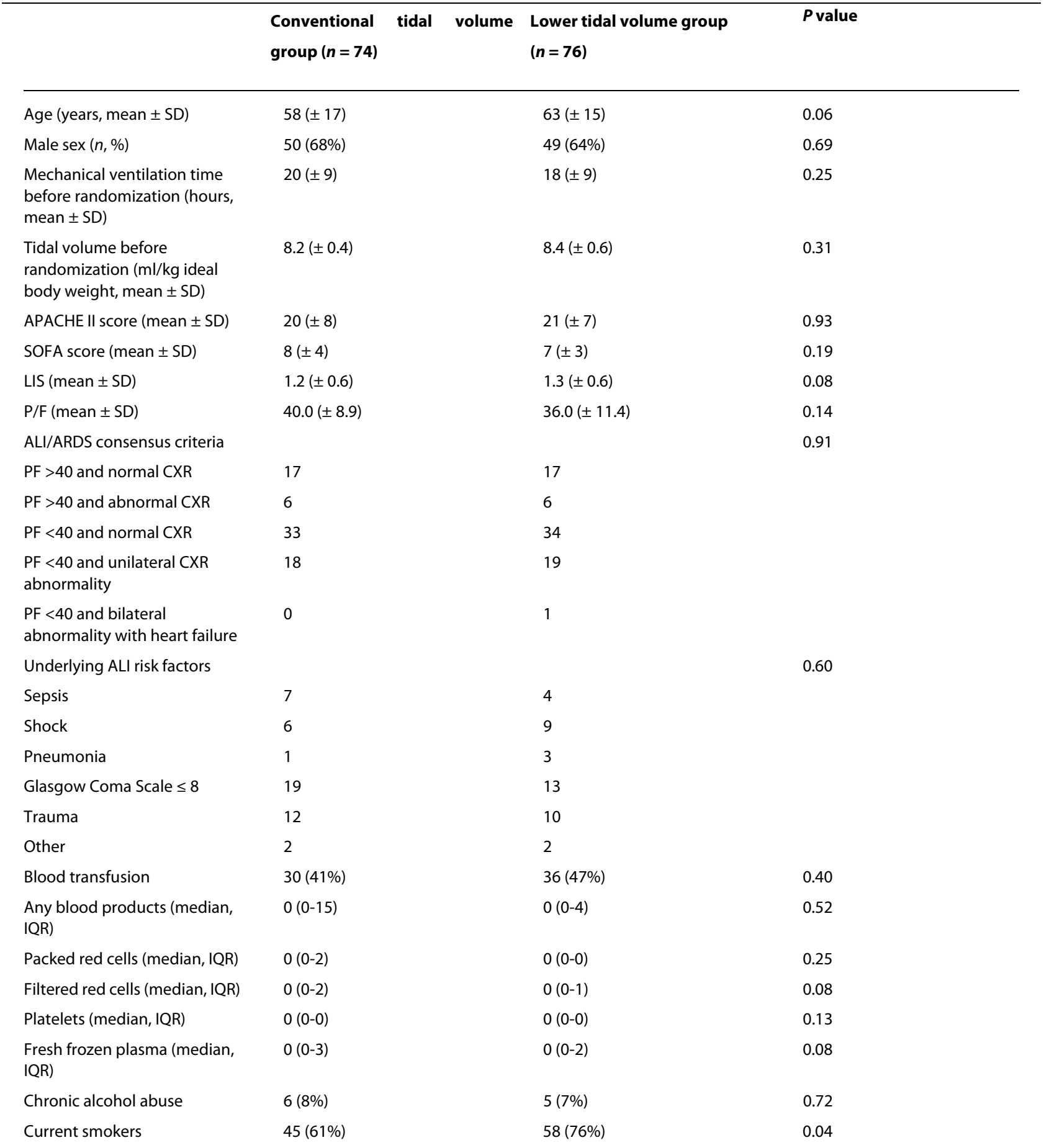

APACHE-II = acute physiology and chronic health evaluation-II; SOFA = sequential organ failure assessment; $\mathrm{LIS}=$ lung injury score. $\mathrm{PF}=\mathrm{PaO}{ }_{2}$ to $\mathrm{FiO}_{2}$ ratio. Other $\mathrm{ALI}$ risk factors: aspiration pneumonitis, pancreatitis, massive blood transfusion, drug overdose. The number of blood products is expressed as median with interquartile range per patient with at least one product. 


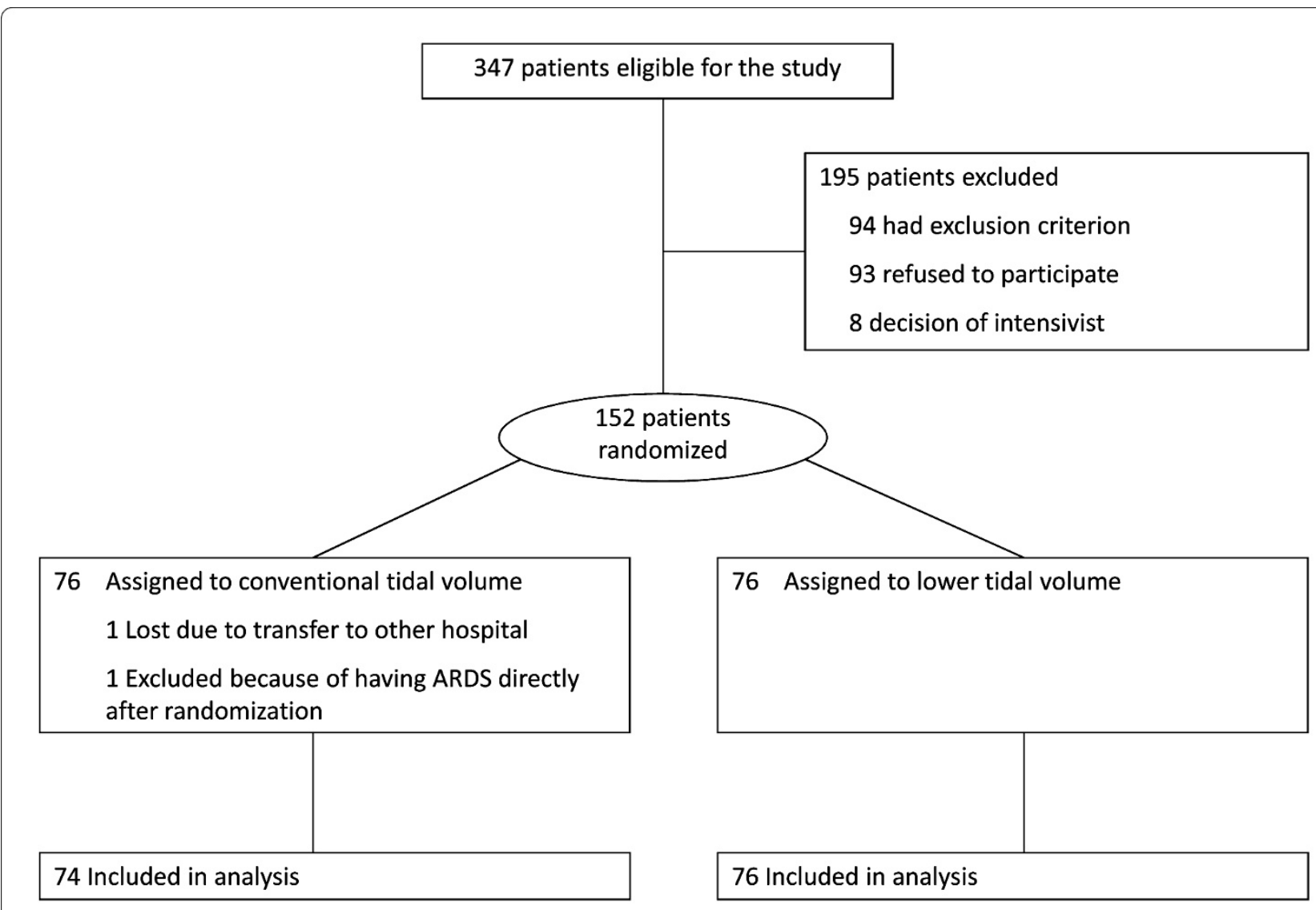

Figure 1 Flow diagram summarizing inclusion, allocation, and analysis. 347 patients were eligible for the study; 94 patients were excluded because of participation in another clinical trial $(n=49)$, use of immunosuppressive agents $(n=22)$, chronic obstructive pulmonary disease ( $n=11)$, prior pneumectomy or lobectomy $(n=5)$, interstitial lung disease $(n=4)$, and pulmonary thromboembolism $(n=3)$; 93 patients refused informed consent, and in eight patients, participation in the trial was denied by the attending physician.

sure $\left(21.6 \pm 7.0 \mathrm{~cm} \mathrm{H}_{2} \mathrm{O}\right.$ versus $24.6 \pm 6.7 \mathrm{~cm} \mathrm{H}_{2} \mathrm{O} ; P=$ 0.009). Both remained lower during the study period (Figure 2). Minute ventilation was comparable at baseline and remained comparable during the study period in both study groups. Respiratory rate was higher at baseline and remained higher in the lower-tidal-volume group $(P<$ $0.001)$.

No differences were observed in the static compliance (Figure 2), blood-gas analysis data, and P/F between the study groups (Figure 3). However, a trend toward a difference in the oxygenation index after 4 days was noted between study groups $(P=0.06)$, and LIS significantly increased after 4 days in the conventional-tidal-volume, whereas it decreased in the lower-tidal-volume group (linear mixed models, interaction time, and group, $P=$ 0.003).

\section{Cytokine levels}

Baseline lavage-fluid levels of TNF- $\alpha$ and IL- $1 \beta$ were comparable in both study groups; baseline lavage-fluid levels of IL-6 were higher in the conventional group, although statistical significance was not reached ((384 (67 to 1,136$) \mathrm{pg} / \mathrm{ml}$ versus $112(20$ to 548$) \mathrm{pg} / \mathrm{ml} ; P=0.07)$ ) (Figure 4). Lavage-fluid levels of cytokines remained comparable over time in both study groups. Baseline plasma IL-6 levels were comparable in both study groups ((50 (21 to 122$) \mathrm{ng} / \mathrm{ml}$ versus 51 (20 to 182$) \mathrm{ng} / \mathrm{ml}$ in the conventional- and lower-tidal-volume groups, respectively; $P=0.74)$ ). In the conventional-tidal-volume group, plasma IL-6 levels decreased after 4 days ((21 (9 to 99) ng/ $\mathrm{ml})$ ), but the decrease over time was more pronounced in the lower-tidal-volume group ((11 (5 to 20$) \mathrm{ng} / \mathrm{ml} ; P=$ $0.01)$ ).

As compared with patients in whom lung injury did not develop, patients in whom lung injury did develop had significantly higher baseline lavage-fluid levels of IL-6 
Table 3: Univariate and multivariate logistic regression analysis of risk factors associated with development of acute lung injury

\begin{tabular}{|c|c|c|c|c|}
\hline & $\begin{array}{l}\text { Lung injury } \\
\qquad(n=12)\end{array}$ & $\begin{array}{l}\text { No lung injury } \\
(n=138)\end{array}$ & $\begin{array}{l}P \text { value } \\
\text { univariate analysis }\end{array}$ & $\begin{array}{l}P \text { value } \\
\text { multivariate analysis }\end{array}$ \\
\hline Age (years) & $63.5(54.3-78.3)$ & $63.0(48.8-74.0)$ & 0.41 & - \\
\hline Male gender $(n, \%)$ & $9(75 \%)$ & $90(65 \%)$ & 0.49 & - \\
\hline APACHE-II score & $19(15-23)$ & $20(15-27)$ & 0.82 & - \\
\hline LIS & $1.5(1.25-2.0)$ & $1.25(0.75-1.75)$ & 0.03 & - \\
\hline ALI-risk factor $(n, \%)$ & $10(83 \%)$ & $82(59 \%)$ & 0.10 & 0.14 \\
\hline $\begin{array}{l}\text { Number of transfused blood products } \\
\text { (median, IQR) }\end{array}$ & $0(0-13)$ & $0(0-2)$ & 0.01 & - \\
\hline Oxygenation Index & $5.2(4.3-6.8)$ & $3.9(2.7-5.5)$ & 0.04 & - \\
\hline $\mathrm{PaO}_{2} / \mathrm{FiO}_{2}$ & $34(26-41)$ & (35 (28-45) & 0.46 & - \\
\hline Conventional-tidal-volume group & $10(83 \%)$ & $64(46 \%)$ & 0.01 & 0.007 \\
\hline PEEP level $\left(\mathrm{cm} \mathrm{H}_{2} \mathrm{O}\right)$ & $10(8-12)$ & $5(5-9)$ & 0.001 & 0.001 \\
\hline $\begin{array}{l}\text { IL-6 level in lavage fluid } \\
\text { (median with IQR, pg/ml) }\end{array}$ & $592(148-1,321)$ & $226(23-765)$ & 0.04 & - \\
\hline IL-6 level in plasma (pg/ml) & $79(25-565)$ & $48(19-145)$ & 0.13 & - \\
\hline $\begin{array}{l}\text { TNF-a level in lavage fluid } \\
\text { (median with IQR, pg/ml) }\end{array}$ & $7.2(1.0-121)$ & $1.3(0-30.6)$ & 0.11 & - \\
\hline $\begin{array}{l}\text { IL- } 1 \beta \text { level in lavage fluid (median with } \\
\text { IQR, } \mathrm{pg} / \mathrm{ml} \text { ) }\end{array}$ & $9.0(0.0-96.7)$ & $42.4(4.2-281)$ & 0.32 & - \\
\hline
\end{tabular}

APACHE-II = acute physiology and chronic health evaluation-II; LIS = lung injury score. Data are expressed as medians with interquartile range or as number with percentage. Underlying risk factors in patients in whom lung injury developed were sepsis $(n=4)$, shock $(n=1)$, trauma $(n$ $=1$ ), drug overdose $(n=1)$, and multiple blood transfusions $(n=1)$ in the conventional-tidal-volume group, and pneumonia $(n=1)$ and shock $(n=1)$ in the lower-tidal-volume group.

((593 (148 to 1,321$] \mathrm{pg} / \mathrm{ml}$ versus 226 (23 to 765$) \mathrm{pg} / \mathrm{ml} ; P$ $=0.04$ ) (Figure 5). Lavage IL-6 levels remained elevated after 4 days. Although baseline plasma levels of IL-6 were comparable between patients in whom lung injury did and did not develop, levels increased after 4 days in patients in whom lung injury developed (Figure 5; $P=$ $0.01)$.

\section{Clinical outcome data}

Twenty-five patients had new or worsening abnormalities on their chest radiographs; 12 patients met the consensus criteria for ALI/ARDS after $1.9 \pm 1.1$ days. Ten of these were randomized to conventional tidal volume, and two, to lower tidal volume mechanical ventilation $\left(P=0.01\right.$; $\chi^{2}$ test), leading to a relative risk of 5.1 (95\% CI, 1.2 to 22.6) for developing lung injury. Patients in whom lung injury developed diverged from patients in whom lung injury did not develop, with respect to minute ventilation, LIS, and static compliance (Figure 6). P/F and oxygenation index changed significantly after 4 days in patients in whom lung injury developed. Underlying risk factors in
ALI/ARDS patients were sepsis $(n=4)$, shock $(n=1)$, trauma $(n=1)$, drug overdose $(n=1)$, and multiple blood transfusions $(n=1)$ in the conventional-tidal-volume group, and pneumonia $(n=1)$ and shock $(n=1)$ in the lower-tidal-volume group.

After 7 days, 13 (25\%) of the surviving patients from the conventional-tidal-volume group and nine $(17 \%)$ from the lower-tidal-volume group were still on the ventilator $(P=0.31)$. After 28 days, the number of ventilator-free days was not different between groups: 24.0 (20.7 to 26.8) days in the conventional-tidal-volume group and 24.0 (21.5 to 25.5$)$ days in the lower-tidal-volume group ( $P=$ $0.88)$. After 28 days, 23 (31\%) patients from the conventional-tidal-volume group and 24 (32\%) patients from the lower-tidal-volume group had died $(P=0.94)$. The Kaplan-Meier curves are shown in Figure 7.

The number of days on which sedatives were used was not significantly different between study groups. In the conventional-tidal-volume group, sedation was used for $1.9 \pm 3.5$ days versus $1.7 \pm 2.2$ days in the lower-tidal-volume group $(P=0.69)$. Neuromuscular blocking drugs 

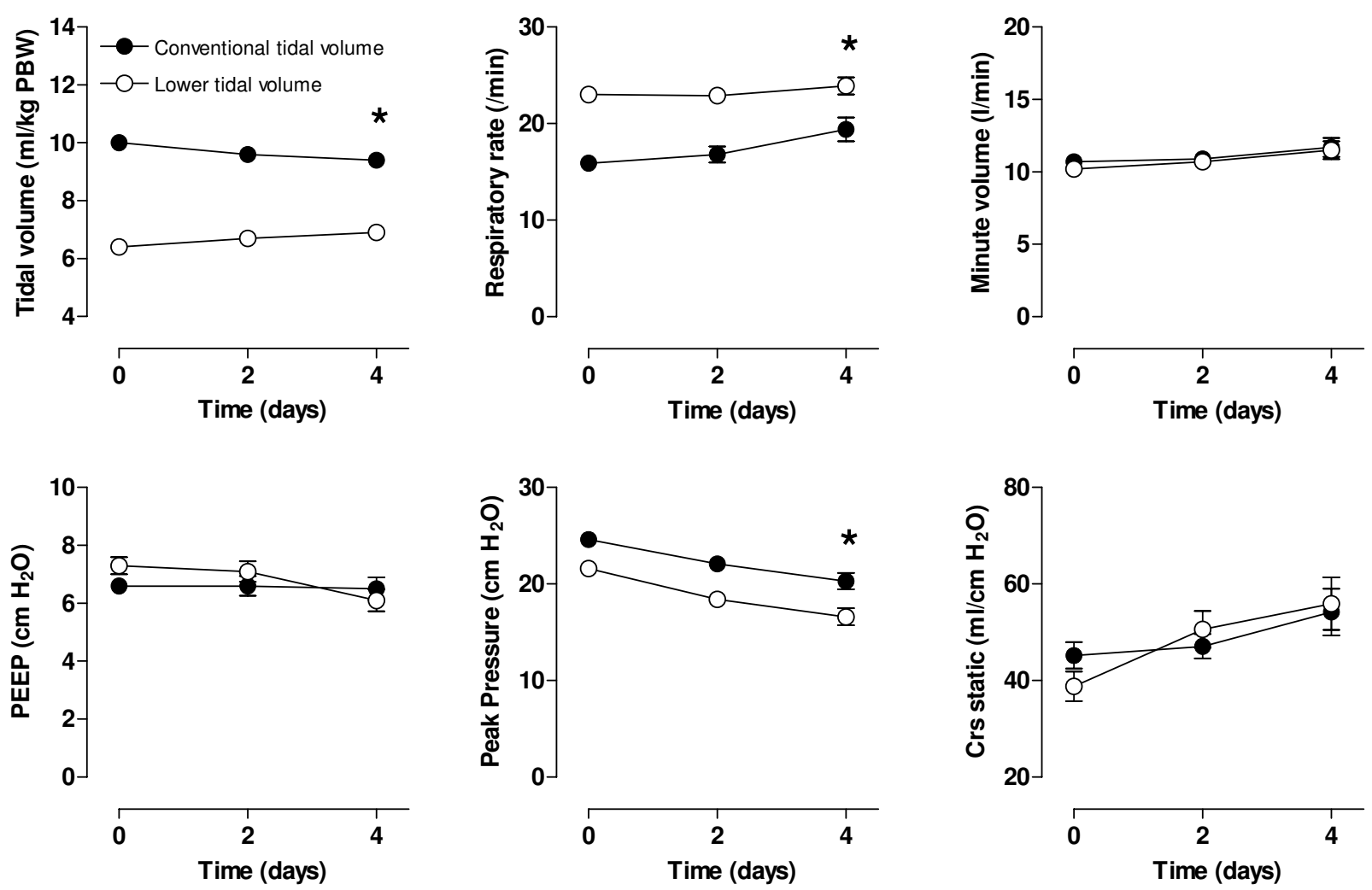

Figure 2 Serial data on mechanical ventilation parameters of patients ventilated with conventional tidal volume (solid circles) or lower tidal volumes (open circles). The number of patients was 74 versus 76 (conventional versus lower tidal volumes), 55 versus 63 , and 34 versus 34 , respectively, at $T=0, T=2$, and $T=4$ days. ${ }^{*} P<0.05$ (Interaction time $\times$ Group).

were used only to facilitate tracheal intubation. The number of days on which vasopressors or inotropic agents were used also was comparable in both study groups (1.8 \pm 3.5 days versus $1.5 \pm 1.9$ days, in conventional- and lower-tidal-volume groups, respectively; $P=0.64$ ).

\section{Univariate and multivariate analysis}

Univariate analysis showed statistical relations between baseline LIS, randomization group, level of PEEP, the number of blood products, ALI/ARDS risk factor, and the baseline IL-6 lavage-fluid level with development of lung injury. Multivariate analysis revealed the randomization group and level of PEEP as independent predictors of lung injury in this study (Table 3).

\section{Discussion}

ALI/ARDS is rarely present at the time of hospital admission but develops over a period of hours to days in patients with predisposing conditions, such as trauma, shock or sepsis, and associated interventions, including mechanical ventilation [24]. Therefore, ALI/ARDS may be viewed as a potentially preventable complication.
Implementation of prevention strategies, such as lungprotective mechanical ventilation with lower tidal volumes lead to a significant decrease in ALI/ARDS and the mortality of mechanically ventilated patients [25]. Although both groups in the present study had a comparable prevalence of risk factors for ALI/ARDS, mechanical ventilation with conventional tidal volumes was associated with a delayed decrease in plasma IL-6 levels and an increased frequency of lung injury after the initiation of mechanical ventilation. The benefit of the use of lower tidal volumes occurred without the need for additional sedation or vasopressor use and was not associated with altered requirements for higher PEEP or additional $\mathrm{FiO}_{2}$.

Conventional mechanical ventilation was accompanied by an altered plasma cytokine profile but not an altered pulmonary cytokine profile. We found plasma IL-6 levels to decrease over time in both groups. The decrease was, however, more pronounced in patients ventilated with lower tidal volumes. This was not accompanied by different cytokine profiles in the lavage fluids. This is in contrast with findings of earlier studies in patients 

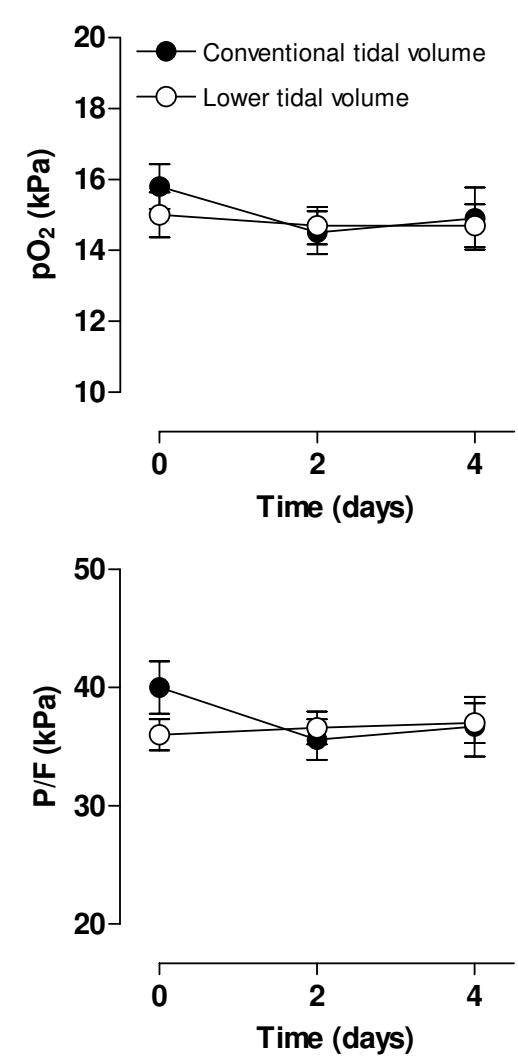
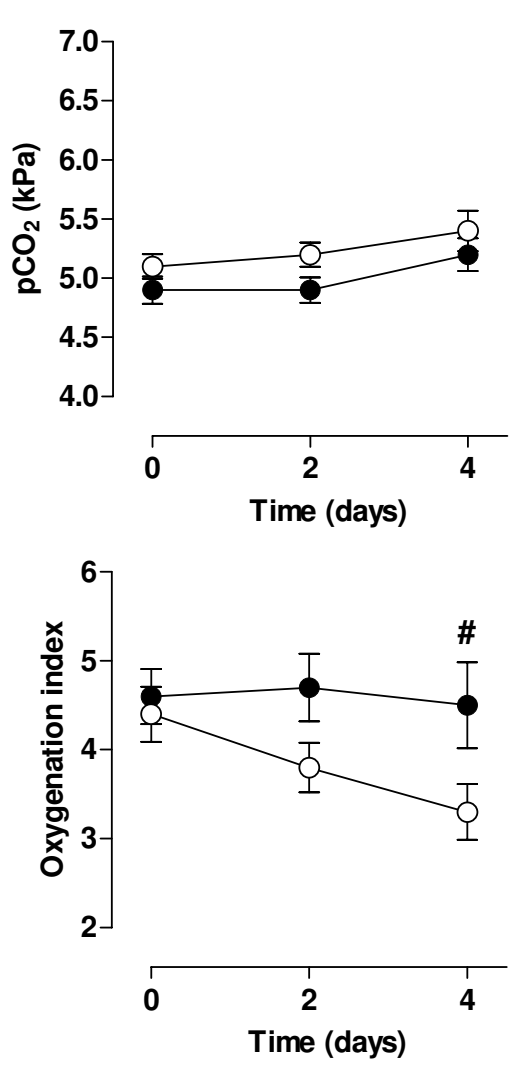
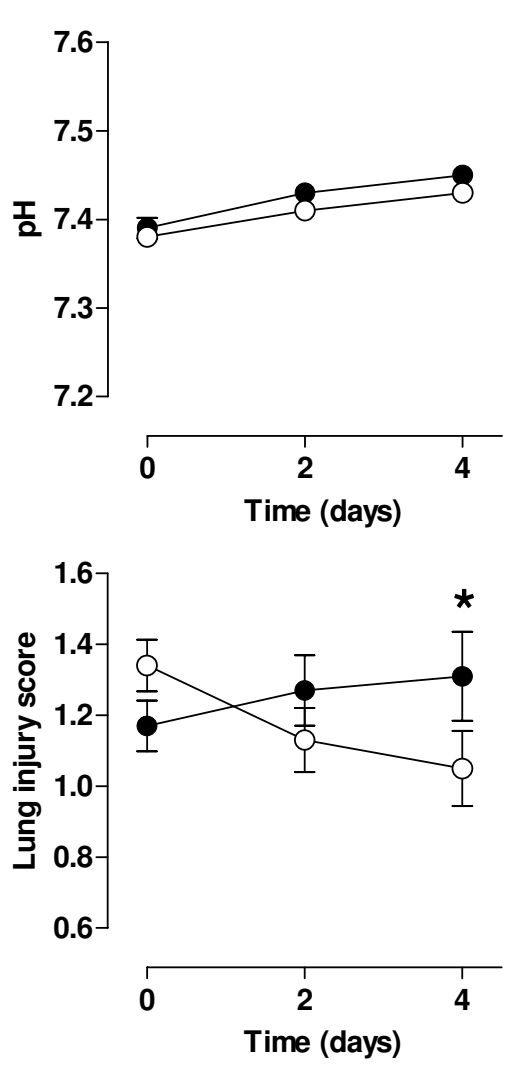

Figure 3 Serial data on respiratory values and lung-injury score of patients ventilated with conventional tidal volume (solid circles) or lower tidal volumes (open circles). $\mathrm{PaO}_{2}$ partial pressure of arterial oxygen; $\mathrm{PaCO}_{2}$ partial pressure of arterial carbon dioxide; $\mathrm{PF}=$ ratio of $\mathrm{PaO} \mathrm{O}_{2}$ to fraction of inspired oxygen; LIS lung injury score. The number of patients was 74 versus 76 (conventional versus lower tidal volumes), 55 versus 63 , and 34 versus 34, respectively, at $T=0, T=2$, and $T=4$ days. ${ }^{*} P<0.05 ;{ }^{*} P=0.06$ (Interaction time $\times$ Group).

undergoing elective surgery. In a recent study, procoagulant changes in lavage fluid of patients with healthy lungs were observed after $5 \mathrm{~h}$ of mechanical ventilation with large tidal volumes [11]. Another recent trial showed increased blood cytokine levels in surgical patients ventilated with conventional tidal volumes compared with those in patients ventilated with lower tidal volumes [12]. The increase in lung-injury score in the conventionaltidal-volume group may not have been reflected by increases in cytokine levels because of a different timing of lavage, deteriorations in $\mathrm{P} / \mathrm{F}$, and changes on chest radiographs. A lavage was performed each second day, but the chest radiographs could be made daily, and bloodgas analyses were routinely performed at least 4 times per day. Moreover, in seven patients, the attending physician reduced the tidal volume size to $6 \mathrm{ml} / \mathrm{kg}$ after the development of lung injury. The study protocol allowed only lung lavage every second day. Lavage procedures were not always performed on the moment of ALI/ARDS diagnosis and before tidal volumes were reduced.
Although we did not observe a general increase in cytokine levels, the mechanism by which mechanical ventilation with conventional tidal volumes leads to fullblown ALI/ARDS in critically ill patients may be as follows. A second-hit model theory can be suggested as a mechanism by which mechanical ventilation may lead to ALI/ARDS. The patients in whom lung injury developed in our study had increased IL-6 levels in their lavage fluid, a higher level of PEEP, and a worse oxygenation index. Although the IL-6 level and oxygenation index were not independent predictors in multivariate analysis, it does show that these patients had some pulmonary inflammation at baseline. It may be speculated that patients with a certain level of inflammation are the patients at risk for ventilator-induced lung injury. Moreover, the baseline level of PEEP was significantly associated with development of lung injury. Larger studies also showed that next to tidal-volume size, risk factors for ALI/ARDS, level of PEEP, and P/F are significant predictors of ALI/ARDS, which is in line with the second-hit model theory $[15,16]$. 

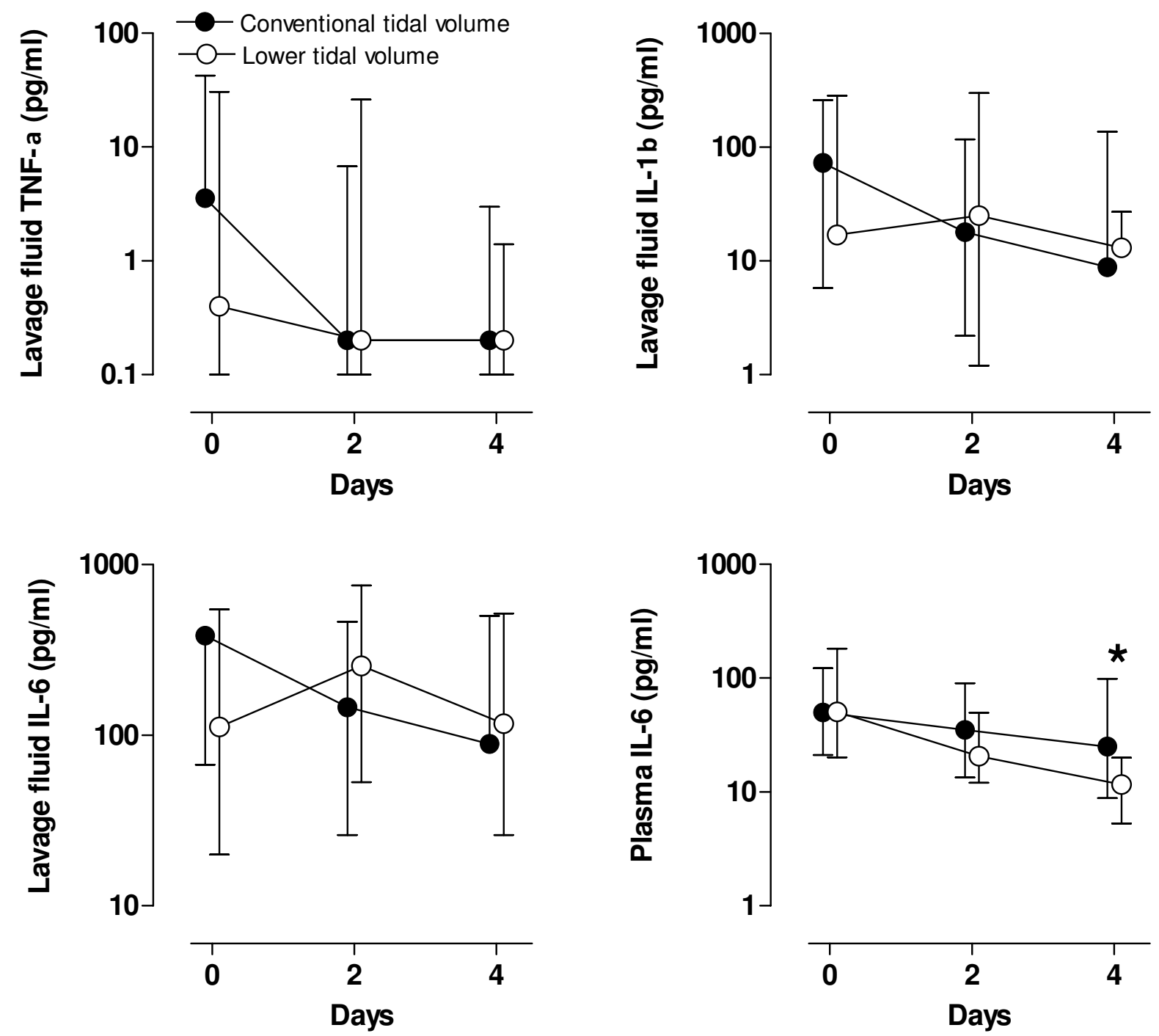

Figure 4 Serial data on cytokine levels in bronchoalveolar lavage fluid of patients ventilated with conventional tidal volume (solid circles) or lower tidal volumes (open circles). TNF- $a=$ tumor necrosis factor- $a ; \mathrm{IL}-1 \beta=$ interleukin- $1 \beta ; \mathrm{IL}-6=$ interleukin- 6 . The number of patients was 74 versus 76 (conventional versus lower tidal volumes), 55 versus 63, and 34 versus 34 , respectively, at $T=0, T=2$, and $T=4$ days. ${ }^{*} P<0.05$; $\# P=0.06$ (Interaction time $\times$ Group).

The study was stopped after the second interim analysis. Interim analyses were not planned at first and were not taken into account on the calculation of sample size, which is a limitation of our study. Although development of lung injury was a secondary end point in our study, the attending physicians of the Academic Medical Center insisted on interim analyses, as they had concerns about the safety of the study. They assumed that the development of lung injury was more frequent with the use of conventional tidal volumes. Therefore, interim analyses were planned halfway and after 150 patients. A stopping boundary was not determined beforehand. As the $P$ value was as low as 0.01 on the second interim analysis, the investigators had no other choice than to stop the trial.

The multivariate analysis showed that tidal volume was an independent predictor of ALI/ARDS development, together with the level of PEEP. Our study was not powered to investigate various risk factors for ALI/ARDS separately in a multivariate model. Therefore, the results of this analysis should be taken with caution. As the level of PEEP may simply have been a marker of disease severity, tidal-volume size may be the only risk factor that can be 

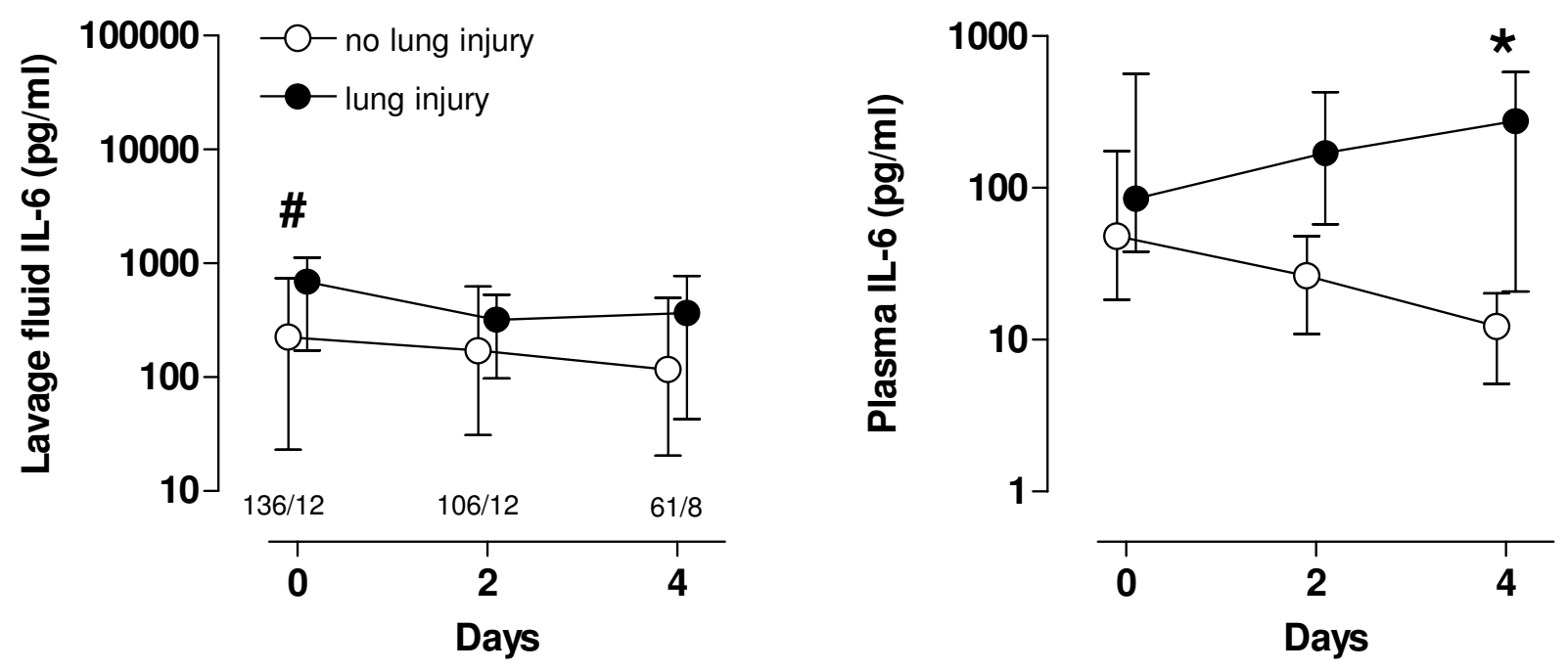

Figure 5 Serial data on cytokine levels in lavage fluid and plasma of patients in whom ALI/ARDS developed (solid circles) and of patients in whom it did not (open circles). The number of patients was 136 versus 12 (no lung injury versus lung injury), 106 versus 12 , and 61 versus 8 , respectively, at $T=0, T=2$, and $T=4$ days. ${ }^{*} P<0.05$.

influenced by the attending physician. Risk factor, oxygenation index, number of transfused blood products, and the baseline lavage-fluid IL-6 level were all associated with lung injury in the univariate analysis and tended to have a significant influence in the multivariate model. As lung injury was a secondary outcome, this study was not powered to investigate all these variables, but larger studies showed that these are significant predictors of ALI/ ARDS $[15,16]$. This is in line with the suggested secondhit model described earlier.

Our findings are in line with earlier reports in patients without ALI/ARDS at the onset of mechanical ventilation $[15,16,23,24]$. The odds ratio of 5.1 for a tidal volume of $10 \mathrm{ml} / \mathrm{kg}$ versus $6 \mathrm{ml} / \mathrm{kg}$ in the present study is in line with earlier findings of an odds ratio of 1.3 for each milliliter above $6 \mathrm{ml} / \mathrm{kg}$ [15]. Although the incidence of development of lung injury in the conventional-tidal-volume group of our study seems quite high (13.5\%), it is still low compared with a comparable cohort of patients without preexisting lung injury in another setting [24]. Of interest, the frequency of ALI/ARDS decreased approximately $65 \%$ after implementation of lung-protective measures, resulting in an incidence of ALI/ARDS comparable to that in our study [25].

The reported mortality rates in ALI/ARDS patients are, however, relatively high, even with lower-tidal-volume mechanical ventilation [3]. We did not find differences in either mortality or the number of ventilator-free days, however. First, our study was not powered to these end points. Second, the protocol required that if lung injury developed, tidal volumes were reduced to $6 \mathrm{ml} / \mathrm{kg}$. This may have underestimated the effect of higher tidal volumes.

Although diagnosing ALI/ARDS is susceptible to subjective interpretation [26], the final decision on the development of lung injury was made by two physicians who were independent of the study. Their judgment was supported by differences in LIS, compliance, and pulmonary and plasma IL-6 levels between patients with and without lung-injury development. Nevertheless, although the physicians were experienced, it may still be possible that some patients who were identified as having ALI/ARDS had hydrostatic pulmonary edema or pulmonary infection and that patients identified as not having ALI/ARDS actually had ALI/ARDS. This may have happened in cardiac-arrest patients, as they are prone to have elevated left atrial pressures and cardiogenic pulmonary edema. Furthermore, as this patient group has a poor prognosis, ALI/ARDS may have been overlooked by the attending physicians. However, all patients with respiratory failure in this group were reviewed by the independent intensivists. As the clinical assessment of ALI/ARDS is hampered by the lack of a gold standard [26], the sensitivity and specificity of any scoring system is moderate at best. The same holds true for the clinical assessment of infection and the identification of patients with ventilator-associated pneumonia [27]. Despite these limitations, we observed an incidence of $8 \%$ in the whole group, which is 

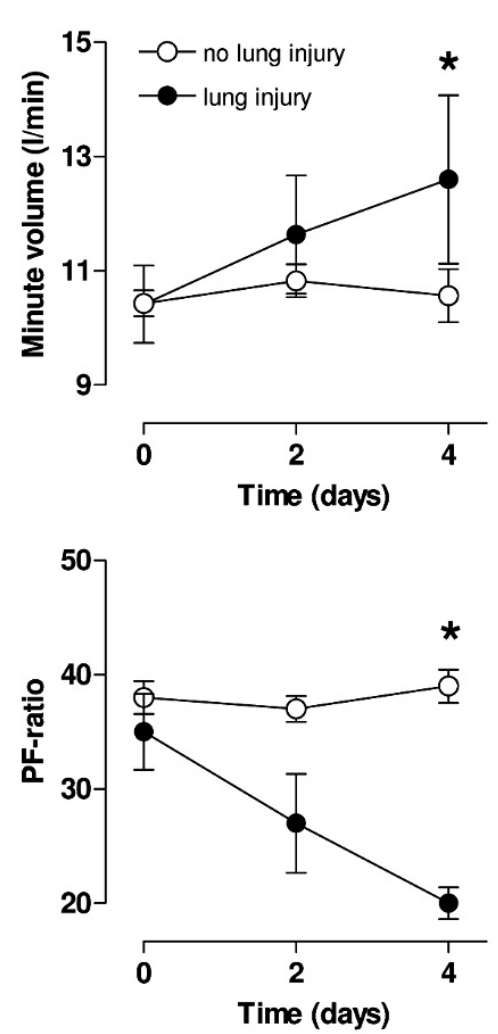
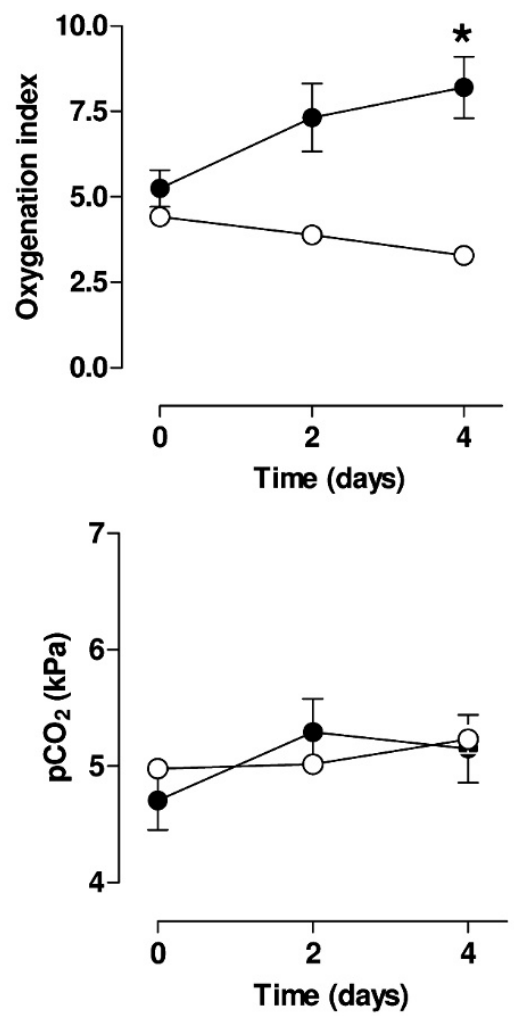
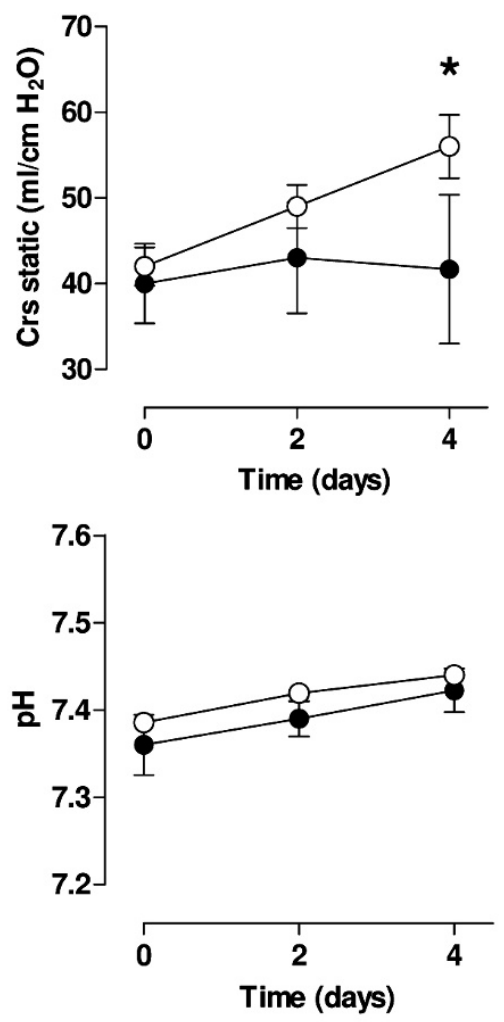

Figure 6 Serial data on minute volume (left graph), oxygenation index (middle graph), and lung compliance (right graph) in patients in whom lung injury developed (solid circles) and in patients in whom it did not (open circles). The number of patients was 136 versus 12 (no ALI/ ARDS versus ALI/ARDS), 106 versus 12, and 61 versus eight, respectively, at $T=0, T=2$, and $T=4$ days. ${ }^{*} P<0.05$.

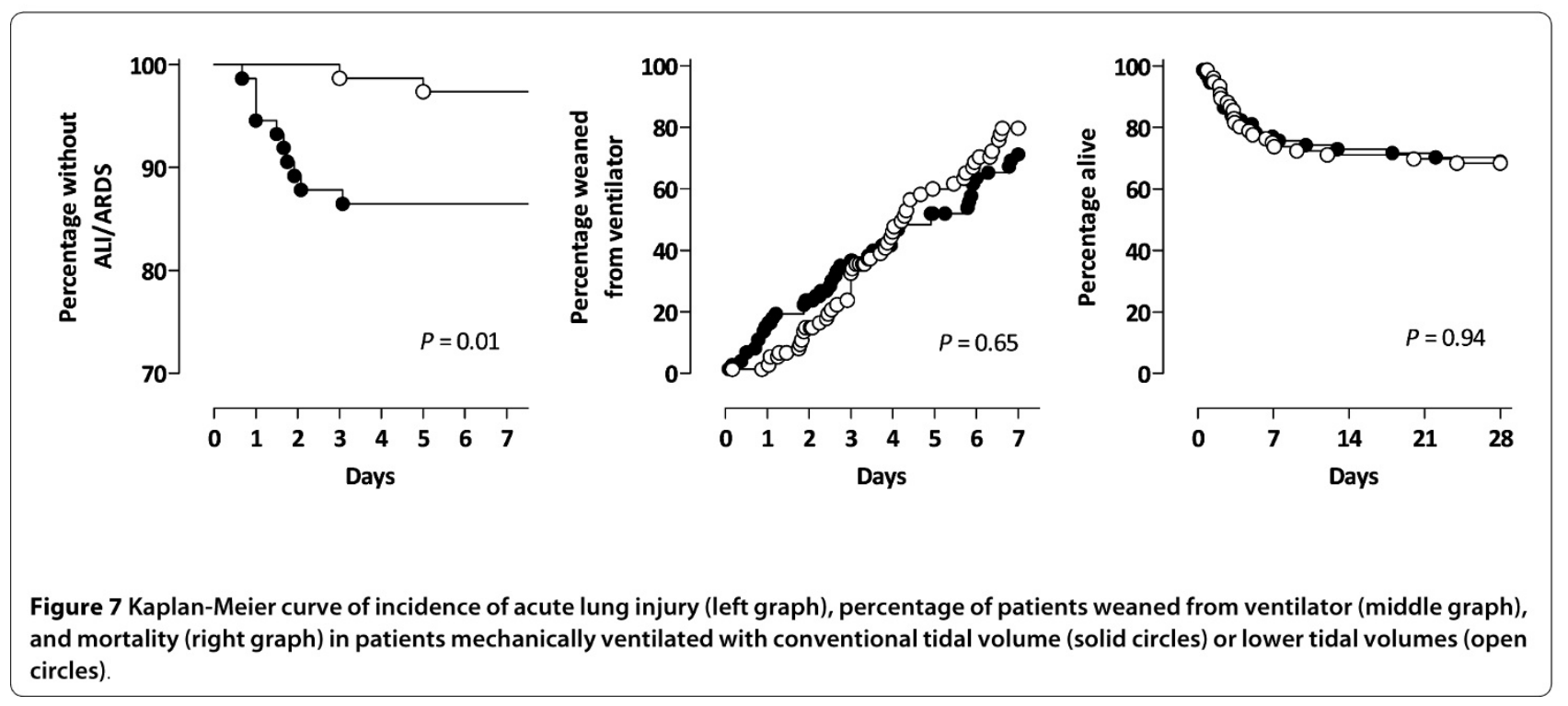


in line with an earlier report of $6 \%$ in a large international cohort study [16].

\section{Conclusions}

Mechanical ventilation with conventional tidal volumes is associated with sustained cytokine production, as measured in plasma. Our data at least suggest that mechanical ventilation with conventional tidal volumes contributes to development of lung injury in patients without ALI at onset of mechanical ventilation. The use of lower tidal volumes did not affect the sedation needs or vasopressor use and was not associated with altered requirements for higher PEEP or additional $\mathrm{FiO}_{2}$. As tidal-volume settings can be determined by physicians, the incidence of this iatrogenic form of lung injury may be reduced. Whether reducing tidal volumes benefits patients with respect to the duration of mechanical ventilation and lower mortality rates remains to be determined in a larger randomized controlled trial.

\section{Key messages}

- Mechanical ventilation with conventional tidal volumes in patients without ALI is associated with sustained cytokine production, as measured in plasma.

- Our data at least suggest that mechanical ventilation with conventional tidal volumes contributes to the development of lung injury in patients without ALI at the onset of mechanical ventilation.

- The use of lower tidal volumes is not associated with higher sedation needs or vasopressor use.

- The use of lower tidal volumes is not associated with requirements for higher PEEP or additional $\mathrm{FiO}_{2}$.

- Larger randomized controlled trials are needed to confirm whether reducing tidal volumes benefits patients with respect to shorter duration of mechanical ventilation and lower mortality rates.

\section{Abbreviations}

ALl: acute lung injury; ARDS: acute respiratory distress syndrome; IL-1 $1 \beta$ : interleukin-1B; IL-6: interleukin-6; LIS: lung injury score; PEEP: positive end-expiratory pressure; TNF-a: tumor necrosis factor-a; SOFA: sequential organ-failure assessment.

\section{Competing interests}

The authors declare that they have no competing interests.

\section{Authors' contributions}

The study was designed by RMD, EKW, LC, and MJS. Acquisition of the data was performed by RMD, AR, EKW, APV, GC, FP, JJH, and MJG. Analysis and interpretation of data was done by RMD, JK, and MJS. The manuscript was drafted by RMD, JK, and MJS. Critical revision of the manuscript for important intellectual content was done by RMD, AR, EKW, AV, GC, FP, JJH, MJG, JK, and MJS. The statistical analysis was done by RMD, JK, and MJS. Final approval of the manuscript was done by RMD, AR, EKW, APV, GC, FP, JJH, MJG, JK, and MJS.

\section{Acknowledgements}

Marcus J. Schultz is supported by The Netherlands Organisation for Health Research and Development (ZonMw) personal grant NWO-VENI 2004 (project no. 016.056.001)

\section{Author Details}

'Department of Intensive Care Medicine, Academic Medical Center, Meibergdreef 9, 1105 AZ, Amsterdam, The Netherlands,

2Department of Internal Medicine, Academic Medical Center, Meibergdreef 9 , 1105 AZ, Amsterdam, The Netherlands,

${ }^{3}$ Department of Intensive Care Medicine, Tergooi Hospitals, Rijksstraatweg 1,

1261 AN, Blaricum, The Netherlands,

4Department of Anesthesiology, Tergooi Hospitals, Rijksstraatweg 1, 1261 AN, Blaricum, The Netherlands,

${ }^{5}$ Department of Anesthesiology, Academic Medical Center, Meibergdreef 9, $1105 \mathrm{AZ}$, Amsterdam, The Netherlands,

${ }^{6}$ Department of Epidemiology and Biostatistics, Academic Medical Center, Meibergdreef 9, 1105 AZ, Amsterdam, The Netherlands and

${ }^{7}$ Laboratory of Experimental Intensive Care and Anesthesiology (L.E.I.C.A), Academic Medical Center, Meibergdreef 9, 1105 AZ, Amsterdam, The

Netherlands

Received: 24 September 2009 Revisions Requested: 12 November 2009

Revised: 22 November 2009 Accepted: 7 January 2010 Published: 7 January 2010

\section{References}

1. Dreyfuss D, Saumon G: Ventilator-induced lung injury: lessons from experimental studies. Am J Respir Crit Care Med 1998, 157:294-323.

2. Carney D, DiRocco J, Nieman G: Dynamic alveolar mechanics and ventilator-induced lung injury. Crit Care Med 2005, 33:S122-128.

3. Plotz FB, Slutsky AS, van Vught AJ, Heijnen CJ: Ventilator-induced lung injury and multiple system organ failure: a critical review of facts and hypotheses. Intensive Care Med 2004, 301865:-1872.

4. Ventilation with lower tidal volumes as compared with traditional tidal volumes for acute lung injury and the acute respiratory distress syndrome: the Acute Respiratory Distress Syndrome Network. NEngl J Med 2000, 342:1301-1308.

5. Amato MB, Barbas CS, Medeiros DM, Magaldi RB, Schettino GP, LorenziFilho G, Kairalla RA, Deheinzelin D, Munoz C, Oliveira R, Takagaki TY, Carvalho CR: Effect of a protective-ventilation strategy on mortality in the acute respiratory distress syndrome. NEng/ J Med 1998, 338:347-354.

6. Villar J, Kacmarek RM, Perez-Mendez L, Aguirre-Jaime A, for the ARIES Network: A high positive end-expiratory pressure, low tidal volume ventilatory strategy improves outcome in persistent acute respiratory distress syndrome: a randomized, controlled trial. Crit Care Med 2006, 34:1311-1318.

7. Dellinger RP, Carlet JM, Masur H, Gerlach H, Calandra T, Cohen J, GeaBanacloche J, Keh D, Marshall JC, Parker MM, Ramsay G, Zimmerman JL, Vincent JL, Levy MM: Surviving Sepsis Campaign Management Guidelines Committee: surviving sepsis campaign: international guidelines for management of severe sepsis and septic shock: 2008. Crit Care Med 2008, 36:296-327.

8. Schultz MJ, Haitsma JJ, Slutsky AS, Gajic O: What tidal volumes should be used in patients without ALI/ARDS? Anesthesiology 2007, 106:1226-1231.

9. Hudson LD, Milberg JA, Anardi D, Maunder RJ: Clinical risks for development of the acute respiratory distress syndrome. Am J Respir Crit Care Med 1995, 151:293-301.

10. Stewart TE, Meade MO, Cook DJ, Granton JT, Hodder RV, Lapinsky SE, Mazer CD, McLean RF, Rogovein TS, Schouten BD, Todd TRJ, Slutsky AS: Evaluation of a ventilation strategy to prevent barotraumas in patients at high risk for acute respiratory distress syndrome. NEngl J Med 1998, 338:355-361.

11. Choi G, Wolthuis EK, Bresser P, Levi M, Poll T van der, Dzoljic M, Vroom MB, Schultz MJ: Mechanical ventilation with lower tidal volumes and positive end-expiratory pressure prevents alveolar coagulation in patients without acute lung injury. Anesthesiology 2006, 105:689-695.

12. Michelet P, D'Journo XB, Roch A, Doddoli C, Marin V, Papazian L, Decamps I, Bregeon F, Thomas P, Auffray JP: Protective ventilation influences systemic inflammation after esophagectomy: a randomized controlled study. Anesthesiology 2006, 105:911-919. 
13. Wrigge $H$, Uhlig U, Baumgarten G, Menzenbach J, Zinserling J, Ernst M, Dromann D, Welz A, Uhlig S, Putensen C: Mechanical ventilation strategies and inflammatory responses to cardiac surgery: a prospective randomized clinical trial. Intensive Care Med 2005, 31:1379-1387.

14. Wrigge H, Uhlig U, Zinserling J, Behrends-Callsen E, Ottersbach G, Fischer $M$, Uhlig S, Putensen C: The effects of different ventilatory settings on pulmonary and systemic inflammatory responses during major surgery. Anesth Analg 2004, 98:775-781.

15. Gajic O, Dara SI, Mendez JL, Adesanya AO, Festic E, Caples SM, Rana R, St Sauver JL, Lymp JF, Afessa B, Hubmayr RD: Ventilator-associated lung injury in patients without acute lung injury at the onset of mechanical ventilation. Crit Care Med 2004, 32:1817-1824.

16. Gajic O, Frutos-Vivar F, Esteban A, Hubmayr RD, Anzueto A: Ventilator settings as a risk factor for acute respiratory distress syndrome in mechanically ventilated patients. Intensive Care Med 2005, 31:922-926.

17. Bernard GR, Artigas A, Brigham KL, Carlet J, Falke K, Hudson L, Lamy M, Legall JR, Morris A, Spragg R: The American-European Consensus Conference on ARDS: definitions, mechanisms, relevant outcomes, and clinical trial coordination. Am J Respir Crit Care Med 1994, 149:818-824.

18. Trachsel D, McCrindle BW, Nakagawa S, Bohn D: Oxygenation index predicts outcome in children with acute hypoxemic respiratory failure. Am J Respir Crit Care Med 2005, 172:206-211.

19. Millo JL, Schultz MJ, Williams C, Weverling GJ, Ringrose T, Mackinlay Cl, Poll T van der, Garrard CS: Compartmentalisation of cytokines and cytokine inhibitors in ventilator-associated pneumonia. Intensive Care Med 2004, 30:68-74.

20. Levy MM, Fink MP, Marshall JC, Abraham E, Angus D, Cook D, Cohen J, Opal SM, Vincent JL, Ramsay G: 2001 SCCM/ESICM/ACCP/ATS/SIS International Sepsis Definitions Conference. Crit Care Med 2003, 31:1250-1256

21. Rello J: Demographics, guidelines, and clinical experience in severe community-acquired pneumonia. Crit Care 2008, 12:S2

22. Thakur L, Kojicic M, Thakur SJ, Pieper MS, Kashyap R, Trillo-Alvarez CA, Javier F, Cartin-Ceba R, Gajic O: Alcohol consumption and development of acute respiratory distress syndrome: a population-based study. Int J Environ Res Public Health 2009, 6:2426-2435.

23. Ranieri VM, Suter PM, Tortorella C, de Tullio R, Dayer JM, Brienza A, Bruno F, Slutsky AS: Effect of mechanical ventilation on inflammatory mediators in patients with acute respiratory distress syndrome: a randomized controlled trial. JAMA 1999, 282:54-61.

24. Iscimen R, Cartin-Ceba R, Yilmaz M, Khan H, Hubmayr RD, Afessa B, Gajic O: Risk factors for the development of acute lung injury in patients with septic shock: an observational cohort study. Crit Care Med 2008, 36:1518-1522

25. Yilmaz M, Keegan MT, Iscimen R, Afessa B, Buck CF, Hubmayr RD, Gajic O: Toward the prevention of acute lung injury: protocol-guided limitation of large tidal volume ventilation and inappropriate transfusion. Crit Care Med 2007, 35:1660-1666.

26. Ferguson ND, Frutos-Vivar F, Esteban A, Fernández-Segoviano P, Aramburu JA, Nájera L, Stewart TE: Acute respiratory distress syndrome: underrecognition by clinicians and diagnostic accuracy of three clinical definitions. Crit Care Med 2005, 33:2228-2234.

27. Fartoukh M, Maitre B, Honore S, Cerf C, Zahar JR, Brun-Buisson C Diagnosing pneumonia during mechanical ventilation: the clinical pulmonary infection score revisited. Am J Respir Crit Care Med 2003, 168:173-179.

doi: $10.1186 /$ cc8230

Cite this article as: Determann et al., Ventilation with lower tidal volumes as compared with conventional tidal volumes for patients without acute lung injury: a preventive randomized controlled trial Critical Care 2010, 14:R1

\section{Submit your next manuscript to BioMed Central} and take full advantage of:

- Convenient online submission

- Thorough peer review

- No space constraints or color figure charges

- Immediate publication on acceptance

- Inclusion in PubMed, CAS, Scopus and Google Scholar

- Research which is freely available for redistribution

Submit your manuscript at www.biomedcentral.com/submit
C BioMed Central 